\title{
Association between folate metabolism-related polymorphisms and colorectal cancer risk
}

\author{
JONG WOO KIM ${ }^{1 *}$, YOUNG JOO JEON ${ }^{2 *}$, MOON JU JANG $^{3}, \mathrm{JUNG}^{2} \mathrm{KIM}^{2}$, SO YOUNG CHONG ${ }^{3}$, \\ KWANG HYUN KO ${ }^{3}$, SEONG GYU HWANG ${ }^{2,3}$, DOYEUN OH ${ }^{2,3}$, $\mathrm{JISU} \mathrm{OH}^{3}$ and NAM KEUN KIM ${ }^{2}$ \\ ${ }^{1}$ Department of Surgery; ${ }^{2}$ Institute for Clinical Research, School of Medicine; ${ }^{3}$ Department of Internal Medicine, \\ CHA Bundang Medical Center, CHA University, Seongnam, Gyeonggi 463-712, Republic of Korea
}

Received September 8, 2014; Accepted February 11, 2015

DOI: $10.3892 / \mathrm{mco} .2015 .520$

\begin{abstract}
Folate has essential roles in DNA synthesis, repair and methylation. Folate metabolism-related gene variants may modulate the levels of this vitamin and affect the cancer risk. Thus, whether these polymorphisms play an important role in carcinogenesis, particularly colorectal cancer (CRC) development, has been a subject interest. The present study investigated the association between polymorphisms in the methylenetetrahydrofolate reductase (MTHFR), thymidylate synthase $(T S)$ and the reduced folate carrier 1 ( $R F C 1)$ genes and CRC risk. Polymorphisms in MTHFR (677C $>\mathrm{T}$ and 1298A >C), TS [1494del6 and the TS enhancer region (TSER)] and $R F C 1 \quad(-43 \mathrm{~T}>\mathrm{C}, 80 \mathrm{G}>\mathrm{A}$ and $696 \mathrm{C}>\mathrm{T})$ were characterized using polymerase chain reaction-restriction fragment length polymorphism in $477 \mathrm{CRC}$ cases and 514 controls. Although no polymorphisms were significantly associated with the CRC risk in the overall sample, significant associations between folate metabolism-related polymorphisms and CRC risk were identified in the stratified analyses. The MTHFR 677CT/1298AC and MTHFR 1298AC+CC/TSER 2R3R genotypes in the presence of plasma folate levels $\leq 4.12 \mathrm{ng} / \mathrm{ml}$ were associated with significantly increased CRC risk. In addition, individuals with the MTHFR 677TT/TSER 3R3R or MTHFR 677/TSER 3R3R/TS 1494 0bp6bp+6bp6bp genotypes and diabetes mellitus (DM) were at an increased risk for CRC. Therefore, the data suggest that i) MTHFR polymorphisms combined with low plasma folate levels and ii) polymorphisms in folate metabolism-related
\end{abstract}

Correspondence to: Professor Nam Keun Kim, Institute for Clinical Research, School of Medicine, CHA University, 351 Yatap-dong, Seongnam, Gyeonggi 463-712, Republic of Korea E-mail:nkkim@cha.ac.kr; namkkim@naver.com

Professor Jisu Oh, Department of Internal Medicine, CHA Bundang Medical Center, CHA University, 351 Yatap-dong, Seongnam, Gyeonggi 463-712, Republic of Korea

E-mail: fascia5@chamc.co.kr

*Contributed equally

Key words: folate, methylenetetrahydrofolate reductase, thymidylate synthase, reduced folate carrier 1 , colorectal cancer genes combined with metabolic syndrome risk factors (hypertension and DM) increase the odds of developing CRC.

\section{Introduction}

Folate is important for cell division and homeostasis due to its essential role in the synthesis of S-adenosyl-methionine, the methyl donor required for all methylation reactions in the cell. In addition to its function in cell homeostasis, folate has been hypothesized to play a role in carcinogenesis, particularly in colorectal cancer (CRC) development. Several mechanisms may underlie folate-deficiency mediated CRC, including DNA strand breaks, aberrant DNA methylation and impaired DNA repair. Thus, folate has been proposed as a possible candidate nutrient for CRC prevention. Genetic variants in folate metabolism-related genes may modulate levels of this vitamin and influence carcinogenesis risk. Thus far, several epidemiological studies have demonstrated that folate is one determinant of the CRC risk.

The effect of several polymorphic genes involved in folate metabolism, including methylenetetrahydrofolate reductase (MTHFR), thymidylate synthase (TS) and reduced folate carrier 1 (RFCl), on CRC risk has been investigated. MTHFR is a key enzyme in folate metabolism and DNA synthesis, as it catalyzes the reduction of 5,10-methylenetetrahydrofolate to 5-methyltetrahydrofolate; the latter is the methyl donor for the conversion of homocysteine to methionine, while the former and its derivatives, are essential cofactors for thymidylate and de novo purine synthesis (1). The $2.2 \mathrm{~kb}$ gene encoding MTHFR is located on chromosome 1 and includes 11 exons. Despite the fact that several MTHFR polymorphisms have been identified, only two, $677 \mathrm{C}>\mathrm{T}$ and $1298 \mathrm{~A}>\mathrm{C}$, have been investigated in depth. The MTHFR C-to-T transition at nucleotide position 677 in exon 4 generates an alanine-to-valine substitution at amino acid 222. This substitution lies at the binding site for flavin adenine dinucleotide, an important cofactor for MTHFR. The A-to-C transversion at nucleotide 1298 (in exon 7) results in the substitution of glutamate for alanine at codon 429, which represents the S-adenosylmethionine regulatory domain of MTHFR (2). These two polymorphisms produce a thermolabile enzyme with reduced functional activity, which results in an altered intracellular distribution of folate substrates (3). A previous meta-analysis reported an association between the 
677C $>$ T polymorphism and decreased CRC risk, although the A1298C substitution was not associated with the CRC risk (4). However, the link between MTHFR polymorphisms and CRC risk has been inconsistent across populations and the statistical significance of such associations appears to be dependent on folate supplementation status $(4,5)$.

TS is a key rate-limiting enzyme in folate metabolism, catalyzing the conversion of deoxyuridine monophosphate to deoxythymidine monophosphate (dTMP). This conversion is indispensable for the production of thymine, a nucleotide required for DNA synthesis and repair. Increased TS levels may lead to an accumulation of homocysteine and/or folate deficiency, while low TS levels impair dTMP synthesis. Two functionally important polymorphisms in the TS 5'- and 3'-untranslated regions (UTRs) have been well characterized. The first is a polymorphism that contains either triple $(3 \mathrm{R})$ or double $(2 \mathrm{R})$ repeats of a 28 -base pair (bp) sequence in the TS enhancer region (TSER). The second is a 6-bp insertion/deletion polymorphism in the 3'UTR at position 1494 . The TSER 3R and TS 1494 6-bp insertion alleles are associated with increased TS production $(6,7)$. In addition to MTHFR and TS, $\mathrm{RFCl}$ is also active in folate metabolism, aiding in the active transport of 5-methyltetrahydrofolate from the plasma to the cytosol. Several polymorphisms in the $R F C 1$ gene appear to influence its affinity for folate (8).

Thus, polymorphisms in the folate metabolism-related genes MTHFR, TS and RFCl may result in altered enzymatic activity, potentially affecting CRC risk. However, the associations detected between genetic variants in folate metabolism-related genes and CRC have been inconsistent across populations. This may be due to nutritional and clinical factors that contribute to the function of folate-related genes and CRC risk. Thus far, only small to moderate-sized studies have been conducted in Asian samples, limiting the conclusions that can be drawn. In the present study, polymorphisms in MTHFR [677C >T (rs1801133) and 1298A >C (rs1801131)], TSER (rs34743033), TS 1494del6 (rs16430) and RFC1 [-43T>C (rs1131596), 80G >A (rs1051266) and 696C >T (rs12659)] were analyzed in patients with CRC and healthy controls. The aim was to clarify the role of these polymorphisms in CRC predisposition using stratified analyses.

\section{Materials and methods}

Study population. A case-control study was conducted of 991 individuals. Four hundred and seventy-seven patients diagnosed with CRC at CHA Bundang Medical Center (Seongnam, Korea) were enrolled between June 1996 and January 2009. The study only included CRC patients who had undergone surgical resection with a curative intent and who had histologically-proven adenocarcinoma. Tumor staging of CRCs was performed according to the sixth edition of the American Joint Committee on Cancer staging manual. The control group consisted of 514 randomly selected individuals, following a health screening at CHA Bundang Medical Center. The screening excluded patients with a history of thrombotic diseases or cancer. Patients with a high baseline blood pressure (systolic $\geq 140 \mathrm{mmHg}$ or diastolic $\geq 90 \mathrm{mmHg}$ ) on more than one occasion or a history of antihypertensive medication were classified as having hypertension (HTN). Patients with high fasting plasma glucose ( $\geq 126 \mathrm{mg} / \mathrm{dl})$, who took oral hypoglycemic agents, or with a history of insulin treatment were classified as having diabetes mellitus (DM). All the study subjects were Korean and provided written informed consent. The study protocol was approved by the Institutional Review Board of CHA Bundang Medical Center.

Genetic analysis. DNA was extracted from leukocytes using a G-DEX ${ }^{\mathrm{TM}}$ II Genomic DNA Extraction kit (Intron Biotechnology, Seongnam, Korea) according to the manufacturer's instructions. Nucleotide changes were determined by polymerase chain reaction (PCR)-restriction fragment length polymorphism analyses. Polymorphisms were characterized following the digestion of amplified DNA with the endonucleases HinfI and Fnu4HI for the MTHFR $677 \mathrm{C}>\mathrm{T}$ and $1298 \mathrm{~A}>\mathrm{C}$ polymorphisms. For the $677 \mathrm{C}>\mathrm{T}$ polymorphism, an undigested PCR product (203 bp) indicated the homozygous wild-type genotype, while three bands of 203, 173 and $30 \mathrm{bp}$ indicated the heterozygous genotype and two bands of 173 and $30 \mathrm{bp}$ indicated the homozygous minor variant genotype. For the 1298A $>$ C polymorphism, a single band of $138 \mathrm{bp}$ indicated the homozygous wild-type genotype and two fragments of 119 and $19 \mathrm{bp}$ indicated the homozygous minor variant genotype. The TSER polymorphism was genotyped according to amplified fragment size; $2 \mathrm{R}$ corresponded to a $220 \mathrm{bp}$ amplicon and 3R corresponded to a $248 \mathrm{bp}$ amplicon. The TS 1494del6 polymorphism was characterized using DraI (New England BioLabs, Ipswich, MA, USA) digestion. The 6-bp insertion-containing 158-bp fragment was digested into 70 and 88 bp fragments following DraI digestion, while the 152-bp fragment without the insertion was not digested. The RFC1 $-43 \mathrm{~T}>\mathrm{C}$ polymorphism was characterized by digesting the PCR product with TspRI (New England Biolabs). The $\mathrm{C}$ allele remained uncut (194 bp), whereas the $\mathrm{T}$ allele was cut into two fragments of 57 and $137 \mathrm{bp}$. The RFCl 80G>A polymorphism was analyzed by digesting the PCR product with HaeII (New England Biolabs). The A allele remained uncut (230 bp), whereas the $\mathrm{G}$ allele was cut into two fragments of 126 and $104 \mathrm{bp}$. The RFC1 696C>T polymorphism was analyzed by digesting the PCR products with EcoNI (New England Biolabs). The C allele remained uncut (341 bp), whereas the $\mathrm{T}$ allele was cut into two fragments of 163 and $178 \mathrm{bp}$. Primers and PCR conditions for each polymorphism analysis were as described previously (9-12). Genotyping of the seven sites was confirmed by sequencing $20 \%$ of the samples, chosen randomly. The concordance rate was $100 \%$.

Measurement of plasma homocysteine and folate levels. Levels of plasma homocysteine and folate were measured in study participants after 12 hours of fasting. Homocysteine levels were determined using a fluorescence polarization immunoassay performed with the Abbott IMx analyzer (Abbott Laboratories, Abbott Park, IL, USA). Folate levels were determined using competitive immunoassays performed on the ACS 180 Chemiluminesence System (Bayer Diagnostics, Tarrytown, NY, USA).

Statistical analysis. $\chi^{2}$ tests were used to assess differences between cases and controls with regard to categorical data, while Student's t-tests were employed for continuous data. The 
Table I. Characteristics of colorectal cancer cases and controls.

\begin{tabular}{|c|c|c|c|}
\hline Characteristics & Cases & Controls & P-value ${ }^{a}$ \\
\hline $\mathrm{N}$ & 477 & 514 & \\
\hline Age, years ${ }^{\mathrm{b}}$ & $62.34 \pm 11.86$ & $62.01 \pm 11.91$ & 0.661 \\
\hline Male gender, n (\%) & $276(57.9)$ & $266(51.8)$ & 0.054 \\
\hline Hypertension, n (\%) & $295(61.8)$ & $241(46.9)$ & $<0.001$ \\
\hline Diabetes mellitus, n (\%) & $163(34.2)$ & $77(15.0)$ & $<0.001$ \\
\hline Homocysteine $^{\mathrm{b}}, \mu \mathrm{mol} / \mathrm{l}$ & $10.41 \pm 5.61(n=442)$ & $10.06 \pm 4.21(\mathrm{n}=508)$ & 0.284 \\
\hline Folate $^{\mathrm{b}}, \mathrm{ng} / \mathrm{ml}$ & $7.70 \pm 6.72(n=440)$ & $9.25 \pm 8.33(\mathrm{n}=414)$ & 0.003 \\
\hline Tumor size $\geq 5 \mathrm{~cm}, \mathrm{n}(\%)$ & $278(58.3)$ & - & \\
\hline Lymph node invasion, $\mathrm{n}(\%)$ & $226(47.4)$ & - & \\
\hline \multicolumn{4}{|l|}{ Tumor site, $\mathrm{n}(\%)$} \\
\hline Proximal colon & $156(32.7)$ & - & \\
\hline Distal colon & $112(23.5)$ & - & \\
\hline Mixed colon & $7(1.5)$ & - & \\
\hline Rectum & $197(41.3)$ & - & \\
\hline Unclassified & $5(1.0)$ & & \\
\hline \multicolumn{4}{|l|}{ TNM stage, n (\%) } \\
\hline I & $49(10.3)$ & - & \\
\hline II & $200(41.9)$ & - & \\
\hline III & $191(40.0)$ & - & \\
\hline IV & $37(7.8)$ & - & \\
\hline
\end{tabular}

${ }^{\mathrm{a}} \chi^{2}$ used for categorical data, two-sided t-test used for continuous data; ${ }^{\text {bdata }}$ are mean \pm standard deviation. TNM, tumor-node-metastasis.

association between each genetic polymorphism and CRC risk was estimated using crude odds ratios, adjusted odds ratios (AORs) and a 95\% confidence interval (CI) obtained from multivariate logistic regression models adjusted for age, gender, and HTN and DM status. Analyses were performed using GraphPad Prism 4.0 (GraphPad Software Inc., San Diego, CA, USA), HAPSTAT 3.0 (University of North Carolina, Chapel Hill, NC, USA) and Medcalc version 12.1.1.0 (Medcalc Software, Mariakerke, Belgium). $\mathrm{P}<0.05$ was considered to indicate a statistically significant difference.

\section{Results}

Patient characteristics. Baseline characteristics of the study population are described in Table I. The proportion of males and females was 51.8 and $48.2 \%$ in the control group and 57.9 and $42.1 \%$ in the CRC case group, respectively. The mean age of the control and case groups was 62.01 and 62.34 years, respectively. There were no differences in the gender distribution and age between the two groups $(\mathrm{P}=0.054$ and 0.661 , respectively). Compared to the control group, $\mathrm{CRC}$ cases had a more frequent HTN and DM history $(\mathrm{P}<0.001)$. Among cases, 275 patients $(57.7 \%)$ had colon cancer and 197 patients $(41.3 \%)$ had rectal cancer. The type of cancer for 5 patients was unclassified due to mixed colon-rectal cancer. Pathological staging following curative resection was as follows: 49 (10.3\%) stage I, $200(41.9 \%)$ stage II, $191(40.0 \%)$ stage III and $37(7.8 \%)$ stage IV cancers.

Genotype frequency and association with CRC risk. Hardy-Weinberg test results confirmed that all the genotypes were in equilibrium $(\mathrm{P}>0.05)$. The distribution of genotypes and the AOR for each genetic polymorphism and CRC are shown in Table II. Overall, the distribution of the MTHFR gene polymorphisms between CRC cases and controls was not different. The AOR associated with the MTHFR $1298 \mathrm{CC}$ genotype was elevated but did not reach statistical significance (AOR=2.36; 95\% CI, 0.94-5.97; $\mathrm{P}=0.069)$. No difference in the distribution of TS (TSER and 1494del6) and RFCl (-43T>C, $80 \mathrm{G}>\mathrm{A}$ and $696 \mathrm{C}>\mathrm{T}$ ) genotypes between cases and controls was detected.

Association between MTHFR, TS and RFCl polymorphisms and $C R C$ risk in stratified analyses. Analyses were conducted that were stratified by age, gender, HTN, DM, tumor site, tumor size and lymph node $(\mathrm{L} / \mathrm{N})$ invasion status to evaluate the association between MTHFR, TS and RFC1 polymorphisms and $C R C$ risk under various conditions. The genotype frequencies of MTHFR, TS and RFC1 polymorphisms in these strata are presented in Table III. The MTHFR 1298CC genotype was associated with an increased risk of CRC compared to the $\mathrm{AA}+\mathrm{AC}$ genotypes in subjects $>62$ years $(\mathrm{AOR}=3.65$; 95\% CI, 1.04-12.73; $\mathrm{P}=0.043)$, females $(\mathrm{AOR}=4.62$; $95 \% \mathrm{CI}, 1.20-17.80 ; \mathrm{P}=0.026)$, patients with rectal cancer $(\mathrm{AOR}=3.27 ; 95 \% \mathrm{CI}, 1.13-9.47 ; \mathrm{P}=0.029)$ and patients with large tumors $(\geq 5 \mathrm{~cm})(\mathrm{AOR}=3.63 ; 95 \% \mathrm{CI}, 1.38-9.53 ; \mathrm{P}=0.009)$ (Table III). Similarly, the TSER 2R3R+2R2R genotypes were associated with an increased risk of CRC compared to the 3R3R genotype in patients $>62$ years $(\mathrm{AOR}=1.63 ; 95 \% \mathrm{CI}, 1.09-2.43$; $\mathrm{P}=0.017)$. The $2 \mathrm{R} 2 \mathrm{R}$ genotype was also associated with an increased CRC risk in patients without HTN (AOR=7.36; 95\% CI, 1.44-37.58; $\mathrm{P}=0.016$ ) or $\mathrm{L} / \mathrm{N}$ invasion $(\mathrm{AOR}=2.81$; 
Table II. Prevalence of MTHFR, TS and RFC1 polymorphisms in colorectal cancer cases and controls.

\begin{tabular}{|c|c|c|c|c|}
\hline Genotypes & Controls $(\mathrm{n}=514)$ & Cases $(n=477)$ & $\operatorname{AOR}(95 \% \mathrm{CI})^{\mathrm{a}}$ & P-value \\
\hline \multicolumn{5}{|l|}{ MTHFR $677 \mathrm{C}>\mathrm{T}$} \\
\hline $\mathrm{CC}$ & $172(33.5)$ & $159(33.3)$ & 1.00 (reference) & \\
\hline $\mathrm{CT}$ & $265(51.6)$ & $248(52.0)$ & $0.99(0.75-1.33)$ & 0.971 \\
\hline TT & $77(15.0)$ & $70(14.7)$ & $0.93(0.62-1.40)$ & 0.736 \\
\hline Dominant (CC vs. CT+TT) & & & $0.98(0.74-1.29)$ & 0.882 \\
\hline Recessive (CC+CT vs. TT) & & & $0.93(0.65-1.34)$ & 0.708 \\
\hline HWE P-value & 0.125 & 0.091 & & \\
\hline \multicolumn{5}{|l|}{ MTHFR $1298 \mathrm{~A}>\mathrm{C}$} \\
\hline AA & $364(70.8)$ & $336(70.4)$ & 1.00 (reference) & \\
\hline $\mathrm{AC}$ & $143(27.8)$ & $125(26.2)$ & $0.96(0.72-1.29)$ & 0.784 \\
\hline $\mathrm{CC}$ & $7(1.4)$ & $16(3.4)$ & $2.36(0.94-5.97)$ & 0.069 \\
\hline Dominant (AA vs. AC+CC) & & & $1.03(0.77-1.37)$ & 0.846 \\
\hline Recessive (AA+AC vs. CC) & & & $2.46(0.98-6.20)$ & 0.056 \\
\hline HWE P-value & 0.089 & 0.305 & & \\
\hline \multicolumn{5}{|l|}{ TSER 2R/3R } \\
\hline 3R3R & $357(69.5)$ & $316(66.2)$ & 1.00 (reference) & \\
\hline $2 \mathrm{R} 3 \mathrm{R}$ & $147(28.6)$ & $148(31.0)$ & $1.08(0.81-1.44)$ & 0.579 \\
\hline $2 \mathrm{R} 2 \mathrm{R}$ & $10(1.9)$ & $13(2.7)$ & $1.63(0.69-3.85)$ & 0.270 \\
\hline Dominant (3R3R vs. 2R3R+2R2R) & & & $1.11(0.84-1.47)$ & 0.443 \\
\hline Recessive (3R3R+2R3R vs. 2R2R) & & & $1.56(0.66-3.66)$ & 0.310 \\
\hline HWE P-value & 0.248 & 0.379 & & \\
\hline \multicolumn{5}{|l|}{ TS 1494 0bp/6bp } \\
\hline Obp0bp & $245(47.7)$ & $250(52.4)$ & 1.00 (reference) & \\
\hline 0bp6bp & $225(43.8)$ & $183(38.4)$ & $0.80(0.61-1.06)$ & 0.115 \\
\hline 6bp6bp & $44(8.6)$ & $44(9.2)$ & $1.05(0.66-1.68)$ & 0.840 \\
\hline Dominant (0bp0bp vs. 0bp6bp+6bp6bp) & & & $0.84(0.65-1.09)$ & 0.196 \\
\hline Recessive (0bp0bp+0bp6bp vs. 6bp6bp) & & & $1.16(0.74-1.82)$ & 0.516 \\
\hline HWE P-value & 0.447 & 0.215 & & \\
\hline \multicolumn{5}{|l|}{$R F C 1-43 \mathrm{~T}>\mathrm{C}$} \\
\hline TT & $108(21.0)$ & $102(21.4)$ & 1.00 (reference) & \\
\hline $\mathrm{TC}$ & $266(51.8)$ & $219(45.9)$ & $0.88(0.63-1.25)$ & 0.485 \\
\hline $\mathrm{CC}$ & $140(27.2)$ & $156(32.7)$ & $1.18(0.82-1.70)$ & 0.368 \\
\hline Dominant (TT vs. $\mathrm{TC}+\mathrm{CC}$ ) & & & $0.99(0.72-1.36)$ & 0.946 \\
\hline Recessive (TT+TC vs. CC) & & & $1.28(0.97-1.70)$ & 0.086 \\
\hline HWE P-value & 0.376 & 0.127 & & \\
\hline \multicolumn{5}{|l|}{$R F C 180 \mathrm{G}>\mathrm{A}$} \\
\hline GG & $103(20.0)$ & $96(20.1)$ & 1.00 (reference) & \\
\hline GA & $242(47.1)$ & $218(45.7)$ & $0.98(0.69-1.39)$ & 0.904 \\
\hline AA & $169(32.9)$ & $163(34.2)$ & $1.05(0.73-1.51)$ & 0.775 \\
\hline Dominant (AA vs. AG+GG) & & & $1.01(0.73-1.39)$ & 0.966 \\
\hline Recessive (AA+AG vs. GG) & & & $1.05(0.80-1.39)$ & 0.709 \\
\hline HWE P-value & 0.334 & 0.140 & & \\
\hline \multicolumn{5}{|l|}{$R F C 1696 \mathrm{C}>\mathrm{T}$} \\
\hline $\mathrm{CC}$ & $110(21.4)$ & $105(22.0)$ & 1.00 (reference) & \\
\hline $\mathrm{CT}$ & $262(51.0)$ & $229(48.0)$ & $0.97(0.69-1.36)$ & 0.867 \\
\hline TT & $142(27.6)$ & $143(30.0)$ & $1.07(0.74-1.53)$ & 0.729 \\
\hline Dominant (CC vs. CT+TT) & & & $1.00(0.73-1.37)$ & 0.996 \\
\hline Recessive (CC+CT vs. TT) & & & $1.09(0.82-1.45)$ & 0.562 \\
\hline HWE P-value & 0.595 & 0.462 & & \\
\hline
\end{tabular}

${ }^{a}$ Models adjusted for age, gender, hypertension and diabetes mellitus status. HWE, Hardy-Weinberg equilibrium; MTHFR, methylenetetrahydrofolate reductase; $T S$, thymidylate synthase; TSER, TS enhancer region; $R F C 1$, reduced folate carrier 1; AOR, adjusted odds ratio; CI, confidence interval.

95\% CI, 1.17-6.76; $\mathrm{P}=0.021$ ) (Table III). The RFC1 -43CC genotype was associated with a significantly increased risk of
CRC compared to the TT + TC genotypes in patients without $\mathrm{DM}(\mathrm{AOR}=1.46 ; 95 \% \mathrm{CI}, 1.06-2.02 ; \mathrm{P}=0.020)$, with large 


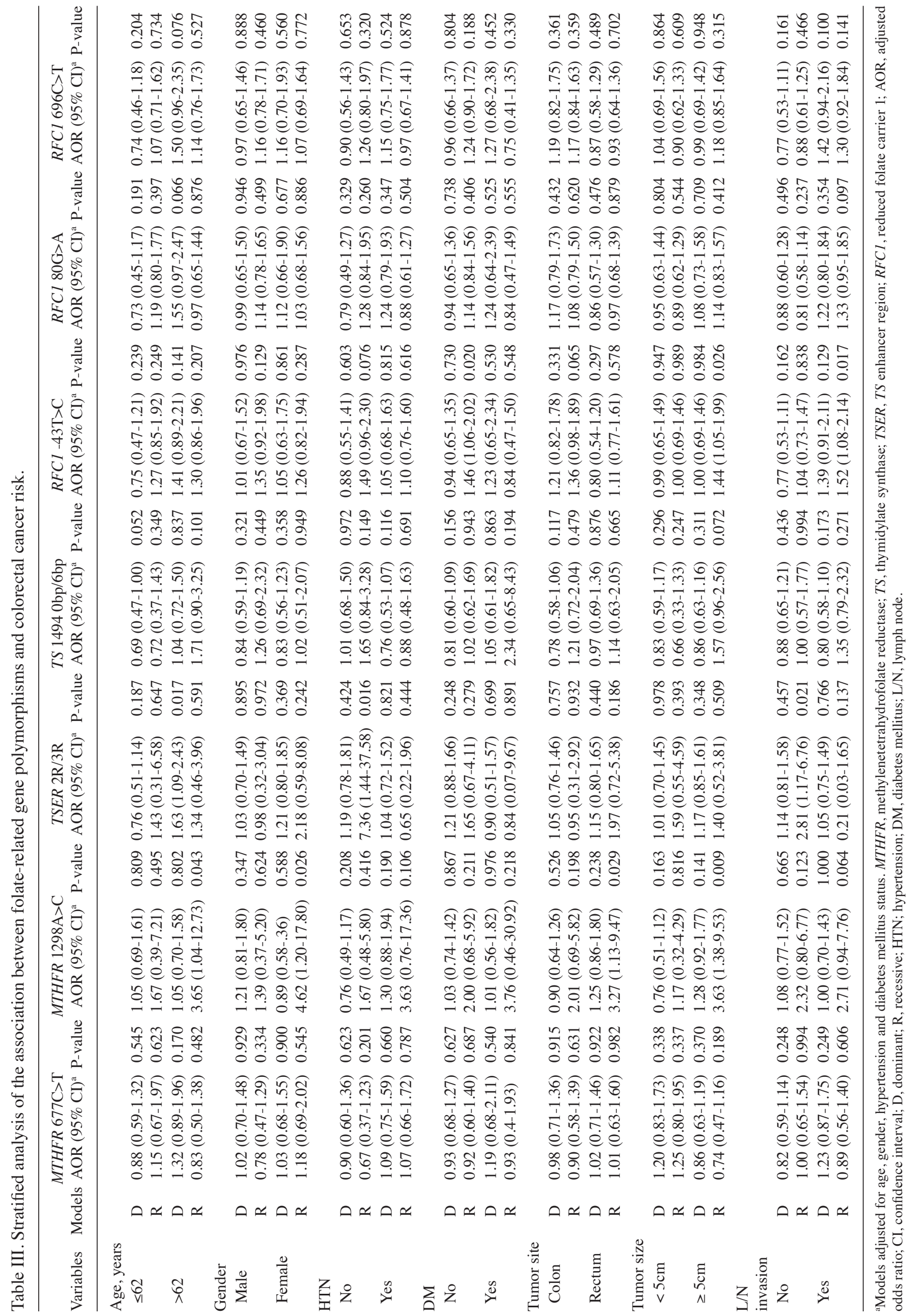


Table IV. Haplotype analysis of $M T H F R, T S$ and $R F C l$ genotypes and colorectal cancer risk.

\begin{tabular}{lccc}
\hline Haplotypes & Controls $(2 \mathrm{n}=1028)$ & Cases $(2 \mathrm{n}=954)$ & OR (95\% CI) \\
\hline MTHFR 677/1298 & & & \\
C-A & $452(44.0)$ & $409(42.9)$ & $0.956(0.801-1.142)$ \\
C-C & $157(15.3)$ & $157(16.5)$ & $1.093(0.859-1.391)$ \\
T-A & $419(40.8)$ & $388(40.7)$ & $0.996(0.833-1.192)$ \\
TSER/TS 1494 & & & 0.650 \\
3R-0bp & $660(64.2)$ & $620(65.0)$ & $1.035(0.861-1.245)$ \\
3R-6bp & $201(19.6)$ & $160(16.8)$ & $0.829(0.659-1.043)$ \\
2R-0bp & $55(5.4)$ & $63(6.6)$ & $1.251(0.861-1.816)$ \\
2R-6bp & $112(10.9)$ & $111(11.6)$ & $1.077(0.815-1.423)$ \\
RFC1 -43/80/696 & & 0.742 \\
T-G-C & $429(41.7)$ & $392(41.1)$ & 0.116 \\
T-G-T & $9(0.9)$ & $2(0.2)$ & 0.255 \\
T-A-C & $35(3.4)$ & $28(2.9)$ & $0.238(0.051-1.104)$ \\
T-A-T & $9(0.9)$ & $1(0.1)$ & $0.858(0.518-1.422)$ \\
C-G-C & $6(0.6)$ & $5(0.5)$ & $0.119(0.015-0.940)$ \\
C-G-T & $4(0.4)$ & $9(0.9)$ & $1.804(0.653-4.985)$ \\
C-A-C & $12(1.2)$ & $507(53.1)$ & $1.349(0.361-5.039)$ \\
C-A-T & $524(51.0)$ & $0.806(0.338-1.923)$ \\
\hline
\end{tabular}

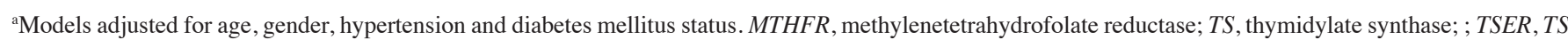
enhancer region; $R F C 1$, reduced folate carrier 1 ; OR, odds ratio; $\mathrm{CI}$, confidence interval.

tumors $(\mathrm{AOR}=1.44 ; 95 \% \mathrm{CI}, 1.05-1.99 ; \mathrm{P}=0.026)$ or with $\mathrm{L} / \mathrm{N}$ invasion $(\mathrm{AOR}=1.52 ; 95 \% \mathrm{CI}, 1.08-2.14 ; \mathrm{P}=0.017)($ Table III $)$.

Haplotype analysis. Haplotype analyses were conducted to evaluate the combined effect of polymorphisms on CRC risk (Table IV). Three haplotypes were constructed using the MTHFR $677 \mathrm{C}>\mathrm{T}$ and $1298 \mathrm{~A}>\mathrm{C}$ polymorphisms, four were constructed using the TSER and TS 1494del6 polymorphisms and eight were constructed using the $R F C l-43 \mathrm{~T}>\mathrm{C}, 80 \mathrm{G}>\mathrm{A}$ and $696 \mathrm{C}>\mathrm{T}$ polymorphisms. Haplotype analysis revealed no significant differences among the $\mathrm{CRC}$ cases and controls with regard to MTHFR and TS polymorphisms. However, the T-A-T $R F C 1$-43/80/696 haplotype was associated with a decreased odds ratio of $\mathrm{CRC}(\mathrm{OR}=0.119 ; 95 \% \mathrm{CI}, 0.015-0.940 ; \mathrm{P}=0.022)$.

Associations between folate metabolism-related polymorphisms and CRC risk, stratified by plasma folate levels, HTN and diabetes status. The combined effects of genotype and environmental factors (HTN, DM and plasma folate levels) on CRC risk were examined. HTN, DM and lower plasma folate levels were associated with increased CRC odds ratios in the present study population. In addition, several synergistic effects between genetic and environmental factors were observed for the CRC risk (Tables V and VI). Individuals with MTHFR 677CT/1298AC (OR=8.99; 95\% CI, 1.76-45.93) or MTHFR 1298AC+CC/TSER 2R3R (OR=10.80; 95\% CI, 1.39-83.76) genotypes and plasma folate levels $\leq 4.12 \mathrm{ng} / \mathrm{ml}$ had significantly higher odds of developing CRC (Table V). In addition, individuals with MTHFR 677TT/TSER 3R3R (OR=5.99; 95\% CI, 1.94-18.45) or MTHFR 677/TSER 3R3R/TS 1494 0bp6bp+6bp6bp (OR=20.13; 95\% CI, 1.76-230.92) genotypes, as well as DM had significantly higher odds of CRC (Table VI).

\section{Discussion}

In the present large hospital-based, case-control study, whether the MTHFR, TS and RFCl polymorphisms were associated with CRC susceptibility was investigated in the Korean population. Overall, the MTHFR, TS and RFCl polymorphisms were not associated with increased CRC risk. However, these polymorphisms were associated with heightened risk in the presence of various clinical or environmental conditions, such as low folate status, HTN or DM.

Numerous studies have investigated the association between the MTHFR gene polymorphisms and CRC susceptibility, but the results have been inconsistent. Previously, a comprehensive meta-analysis demonstrated that the MTHFR $677 \mathrm{~T}$ allele is associated with reduced risk of CRC (13). In addition, Yang et al (14) conducted a meta-analysis incorporating 21 studies of the Asian population and obtained similar results. Although the mechanism responsible for the association between the MTHFR $677 \mathrm{C}>\mathrm{T}$ polymorphism and CRC risk remains unclear, it is possible that MTHFR variants increase the level of 5,10-methylenetetrahydrofolate available for DNA synthesis, thereby protecting against CRC. In contrast to these meta-analysis results, a protective effect associated with the MTHFR $677 \mathrm{C}>\mathrm{T}$ polymorphism was not identified. However, among the 61 studies considered in the comprehensive meta-analysis cited, only 4 showed a protective effect, while 53 did not show any significant association between MTHFR $677 \mathrm{C}>\mathrm{T}$ status and CRC risk. A few studies have also demonstrated an association between MTHFR 1298A $>$ C status and CRC (5). Collectively, the available research suggests that the MTHFR $677 \mathrm{C}>\mathrm{T}$ and $1298 \mathrm{~A}>\mathrm{C}$ polymorphisms have a weak impact on CRC risk, absent consideration of interplay with nutritional or environmental factors. Previously, 
Table V. Adjusted odds ratios for polymorphisms in folate-related genes and colorectal cancer, stratified by plasma folate concentrations ${ }^{\mathrm{a}}$.

\begin{tabular}{|c|c|c|c|c|c|}
\hline \multirow[b]{2}{*}{ Genotypes } & \multicolumn{5}{|c|}{ Plasma folate quintiles, $\mathrm{ng} / \mathrm{ml}$} \\
\hline & $>11$ & $>7.72$ to $\leq 11$ & $>5.76$ to $\leq 7.72$ & $>4.12$ to $\leq 5.76$ & $\leq 4.12$ \\
\hline $\mathrm{N}$ & 170 & 170 & 172 & 170 & 172 \\
\hline Total & 1.00 (reference) & $1.10(0.69-1.73)$ & $1.34(0.85-2.11)$ & $1.54(0.98-2.42)$ & $3.34(2.06-5.43)$ \\
\hline \multicolumn{6}{|l|}{ MTHFR $677 \mathrm{C}>\mathrm{T}$} \\
\hline $\mathrm{CC}$ & 1.00 (reference) & $0.62(0.29-1.32)$ & $1.09(0.50-2.35)$ & $1.12(0.51-2.47)$ & $3.23(1.24-8.39)$ \\
\hline $\mathrm{CT}+\mathrm{TT}$ & 1.00 (reference) & $1.53(0.85-2.78)$ & $1.48(0.83-2.62)$ & $1.85(1.06-3.25)$ & $3.68(2.04-6.63)$ \\
\hline \multicolumn{6}{|l|}{ MTHFR $1298 \mathrm{~A}>\mathrm{C}$} \\
\hline $\mathrm{AA}$ & 1.00 (reference) & $1.25(0.72-2.18)$ & $1.31(0.76-2.25)$ & $1.38(0.81-2.38)$ & $2.69(1.52-4.77)$ \\
\hline $\mathrm{AC}+\mathrm{CC}$ & 1.00 (reference) & $0.67(0.28-1.62)$ & $1.41(0.61-3.28)$ & $1.85(0.77-4.44)$ & $6.10(2.27-16.44)$ \\
\hline \multicolumn{6}{|l|}{ TSER 2R/3R } \\
\hline $3 R 3 R$ & 1.00 (reference) & $1.19(0.68-2.09)$ & $1.11(0.63-1.95)$ & $1.62(0.93-2.83)$ & $3.09(1.72-5.55)$ \\
\hline $2 \mathrm{R} 3 \mathrm{R}+2 \mathrm{R} 2 \mathrm{R}$ & 1.00 (reference) & $0.96(0.43-2.16)$ & $1.96(0.90-4.27)$ & $1.38(0.62-3.04)$ & $3.99(1.63-9.74)$ \\
\hline \multicolumn{6}{|l|}{ TS 1494 0bp/6bp } \\
\hline Obp0bp & 1.00 (reference) & $1.32(0.66-2.65)$ & $1.40(0.72-2.69)$ & $1.35(0.72-2.54)$ & $3.57(1.71-7.47)$ \\
\hline 0bp6bp+6bp6bp & 1.00 (reference) & $1.01(0.54-1.90)$ & $1.33(0.70-2.51)$ & $1.65(0.85-3.21)$ & $3.45(1.77-6.73)$ \\
\hline \multicolumn{6}{|l|}{ MTHFR 677/1298 } \\
\hline $\mathrm{CC} / \mathrm{AA}$ & 1.00 (reference) & $0.50(0.16-1.55)$ & $0.47(0.13-1.66)$ & $0.35(0.09-1.27)$ & $2.59(0.51-13.12)$ \\
\hline $\mathrm{CC} / \mathrm{AC}+\mathrm{CC}$ & 1.00 (reference) & $0.59(0.19-1.87)$ & $1.90(0.61-5.90)$ & $2.69(0.85-8.56)$ & $4.79(1.34-17.13)$ \\
\hline $\mathrm{CT}+\mathrm{TT} / \mathrm{AA}$ & 1.00 (reference) & $1.70(0.87-3.33)$ & $1.56(0.82-2.98)$ & $1.95(1.04-3.64)$ & $3.13(1.64-5.98)$ \\
\hline $\mathrm{CT} / \mathrm{AC}$ & 1.00 (reference) & $0.92(0.22-3.92)$ & $1.08(0.27-4.34)$ & $1.14(0.27-4.82)$ & $8.99(1.76-45.93)$ \\
\hline \multicolumn{6}{|l|}{ MTHFR 677/TSER } \\
\hline $\mathrm{CC} / 3 \mathrm{R} 3 \mathrm{R}$ & 1.00 (reference) & $0.88(0.35-2.18)$ & $0.65(0.24-1.78)$ & $1.91(0.70-5.19)$ & $2.19(0.73-6.62)$ \\
\hline $\mathrm{CC} / 2 \mathrm{R} 3 \mathrm{R}+2 \mathrm{R} 2 \mathrm{R}$ & 1.00 (reference) & $0.22(0.03-1.66)$ & $2.50(0.60-10.49)$ & $0.40(0.09-1.81)$ & $10.35(0.83-129.72)$ \\
\hline $\mathrm{CT}+\mathrm{TT} / 3 \mathrm{R} 3 \mathrm{R}$ & 1.00 (reference) & $1.49(0.71-3.12)$ & $1.34(0.66-2.74)$ & $1.60(0.81-3.20)$ & $3.66(1.78-7.50)$ \\
\hline $\mathrm{CT}+\mathrm{TT} / 2 \mathrm{R} 3 \mathrm{R}+2 \mathrm{R} 2 \mathrm{R}$ & 1.00 (reference) & $1.67(0.59-4.70)$ & $1.83(0.67-4.99)$ & $2.71(0.97-7.61)$ & $4.96(1.51-16.28)$ \\
\hline \multicolumn{6}{|l|}{ MTHFR 1298/TSER } \\
\hline $\mathrm{AA} / 3 \mathrm{R} 3 \mathrm{R}$ & 1.00 (reference) & $1.64(0.82-3.27)$ & $1.24(0.62-2.49)$ & $1.48(0.75-2.89)$ & $2.86(1.42-5.73)$ \\
\hline $\mathrm{AA} / 2 \mathrm{R} 3 \mathrm{R}+2 \mathrm{R} 2 \mathrm{R}$ & 1.00 (reference) & $0.82(0.30-2.25)$ & $1.60(0.64-3.97)$ & $1.19(0.47-3.04)$ & $2.54(0.87-7.39)$ \\
\hline $\mathrm{AC}+\mathrm{CC} / 3 \mathrm{R} 3 \mathrm{R}$ & 1.00 (reference) & $0.55(0.18-1.67)$ & $1.02(0.36-2.84)$ & $2.03(0.70-5.86)$ & $4.48(1.37-14.70)$ \\
\hline $\mathrm{AC}+\mathrm{CC} / 2 \mathrm{R} 3 \mathrm{R}$ & 1.00 (reference) & $0.89(0.17-4.65)$ & $6.97(0.89-54.71)$ & $1.57(0.28-8.86)$ & $10.80(1.39-83.76)$ \\
\hline \multicolumn{6}{|l|}{ MTHFR 1298/TS 1494} \\
\hline AA/0bp0bp & 1.00 (reference) & $1.47(0.65-3.33)$ & $1.35(0.62-2.95)$ & $1.35(0.63-2.88)$ & $3.47(1.44-8.34)$ \\
\hline AA/0bp6bp+6bp6bp & 1.00 (reference) & $1.13(0.52-2.46)$ & $1.30(0.59-2.87)$ & $1.32(0.60-2.93)$ & $2.23(0.99-5.04)$ \\
\hline $\mathrm{AC}+\mathrm{CC} / 0 \mathrm{bp} 0 \mathrm{bp}$ & 1.00 (reference) & $0.81(0.17-3.91)$ & $1.30(0.36-4.68)$ & $1.52(0.44-5.28)$ & $4.24(0.93-19.38)$ \\
\hline $\mathrm{AC}+\mathrm{CC} / 0 \mathrm{bp} 6 \mathrm{bp}+6 \mathrm{bp} 6 \mathrm{bp}$ & 1.00 (reference) & $0.69(0.21-2.25)$ & $1.45(0.45-4.62)$ & $2.48(0.61-10.04)$ & $8.85(2.23-35.16)$ \\
\hline \multicolumn{6}{|l|}{ TSER/TS 1494} \\
\hline 3R3R/0bp0bp & 1.00 (reference) & $1.79(0.79-4.07)$ & $1.43(0.66-3.07)$ & $1.76(0.85-3.65)$ & $4.07(1.75-9.47)$ \\
\hline 3R3R/0bp6bp+6bp6bp & 1.00 (reference) & $0.99(0.42-2.38)$ & $0.82(0.34-1.98)$ & $1.22(0.47-3.18)$ & $2.70(1.07-6.79)$ \\
\hline $2 \mathrm{R} 3 \mathrm{R}+2 \mathrm{R} 2 \mathrm{R} / 0 \mathrm{bp} 0 \mathrm{bp}$ & 1.00 (reference) & $0.63(0.11-3.69)$ & $2.79(0.53-14.67)$ & $0.42(0.08-2.11)$ & $2.79(0.40-19.67)$ \\
\hline $2 \mathrm{R} 3 \mathrm{R}+2 \mathrm{R} 2 \mathrm{R} / 0 \mathrm{bp} 6 \mathrm{bp}+6 \mathrm{bp} 6 \mathrm{bp}$ & 1.00 (reference) & $1.15(0.44-3.04)$ & $2.38(0.90-6.29)$ & $2.39(0.88-6.47)$ & $4.77(1.64-13.86)$ \\
\hline
\end{tabular}

${ }^{a}$ Models adjusted for age, gender, hypertension, diabetes mellitus and plasma folate concentrations. Results for genetic combinations associated with ORs lower than that found for the total sample in the lowest quintile (folate $\leq 4.12 \mathrm{ng} / \mathrm{ml}$ ) are not shown. MTHFR, methylenetetrahydrofolate reductase; TS, thymidylate synthase; TSER, TS enhancer region; ORs, odds ratios.

Ma et al (15) demonstrated that the association of the MTHFR $677 \mathrm{C}>\mathrm{T}$ polymorphism with the $\mathrm{CRC}$ risk is modulated by diet-supplied methyl levels. This study identified that the protective effect of the polymorphism was absent in subjects with plasma folate levels $\geq 3 \mathrm{ng} / \mathrm{ml}$ or low alcohol consumption (15). Keku et al (5) also reported interactions between folate intake and two MTHFR polymorphisms. In their study, in individuals who have low dietary folate intake, the MTHFR
677CT+TT genotype was associated with an increased CRC risk. Similarly, the MTHFR 1298AA genotype in individuals with low dietary folate intake was associated with significantly increased CRC risk, due to linkage disequilibrium between the MTHFR 677T and 1298A alleles (5). As the present study did not consider the individual participant dietary habits, the impact of folate intake on CRC risk could not be assessed. However, the interplay between MTHFR polymorphisms and 
Table VI. Adjusted odds ratios for polymorphisms in folate-related genes and colorectal cancer, stratified by hypertension and diabetes mellitus status ${ }^{\mathrm{a}}$.

\begin{tabular}{|c|c|c|c|c|}
\hline \multirow[b]{2}{*}{ Genotypes } & \multicolumn{2}{|c|}{ Hypertension } & \multicolumn{2}{|c|}{ Diabetes mellitus } \\
\hline & No & Yes & No & Yes \\
\hline $\mathrm{N}$ & 455 & 536 & 751 & 240 \\
\hline Total & 1.00 (reference) & $1.72(1.31-2.26)$ & 1.00 (reference) & $2.73(2.00-3.73)$ \\
\hline \multicolumn{5}{|l|}{ MTHFR $677 \mathrm{C}>\mathrm{T}$} \\
\hline $\mathrm{CC}+\mathrm{CT}$ & 1.00 (reference) & $1.59(1.19-2.14)$ & 1.00 (reference) & $2.73(1.94-3.83)$ \\
\hline TT & 1.00 (reference) & $2.74(1.30-5.79)$ & 1.00 (reference) & $3.16(1.41-7.12)$ \\
\hline \multicolumn{5}{|l|}{ MTHFR $1298 \mathrm{~A}>\mathrm{C}$} \\
\hline AA & 1.00 (reference) & $1.52(1.10-2.09)$ & 1.00 (reference) & 2.77 (1.91-4.04) \\
\hline $\mathrm{AC}+\mathrm{CC}$ & 1.00 (reference) & $2.52(1.49-4.26)$ & 1.00 (reference) & $2.67(1.51-4.73)$ \\
\hline \multicolumn{5}{|l|}{ TSER 2R/3R } \\
\hline 3R3R & 1.00 (reference) & $1.90(1.36-2.65)$ & 1.00 (reference) & 3.07 (2.07-4.56) \\
\hline $2 \mathrm{R} 3 \mathrm{R}+2 \mathrm{R} 2 \mathrm{R}$ & 1.00 (reference) & $1.41(0.88-2.27)$ & 1.00 (reference) & $2.26(1.35-3.80)$ \\
\hline \multicolumn{5}{|l|}{ TS 1494 0bp/6bp } \\
\hline Obp0bp & 1.00 (reference) & $2.08(1.41-3.06)$ & 1.00 (reference) & $2.50(1.62-3.87)$ \\
\hline 0bp6bp+6bp6bp & 1.00 (reference) & $1.41(0.96-2.08)$ & 1.00 (reference) & $3.10(1.97-4.88)$ \\
\hline \multicolumn{5}{|l|}{ MTHFR 677/1298 } \\
\hline $\mathrm{CC}+\mathrm{CT} / \mathrm{AA}$ & 1.00 (reference) & $1.30(0.90-1.86)$ & 1.00 (reference) & $2.80(1.82-4.30)$ \\
\hline $\mathrm{CC}+\mathrm{CT} / \mathrm{AC}+\mathrm{CC}$ & 1.00 (reference) & $2.52(1.49-4.26)$ & 1.00 (reference) & $2.67(1.51-4.73)$ \\
\hline TT/AA & 1.00 (reference) & $2.74(1.30-5.79)$ & 1.00 (reference) & $3.16(1.41-7.12)$ \\
\hline \multicolumn{5}{|l|}{ MTHFR 677/TSER } \\
\hline $\mathrm{CC}+\mathrm{CT} / 3 \mathrm{R} 3 \mathrm{R}$ & 1.00 (reference) & $1.85(1.30-2.65)$ & 1.00 (reference) & $2.84(1.85-4.35)$ \\
\hline $\mathrm{CC}+\mathrm{CT} / 2 \mathrm{R} 3 \mathrm{R}+2 \mathrm{R} 2 \mathrm{R}$ & 1.00 (reference) & $1.12(0.67-1.89)$ & 1.00 (reference) & $2.57(1.44-4.58)$ \\
\hline TT/3R3R & 1.00 (reference) & $2.04(0.78-5.33)$ & 1.00 (reference) & $5.99(1.94-18.45)$ \\
\hline $\mathrm{TT} / 2 \mathrm{R} 3 \mathrm{R}+2 \mathrm{R} 2 \mathrm{R}$ & 1.00 (reference) & $4.63(1.26-16.96)$ & 1.00 (reference) & $1.60(0.41-6.24)$ \\
\hline \multicolumn{5}{|l|}{ MTHFR 677/TS 1494} \\
\hline $\mathrm{CC}+\mathrm{CT} / 0 \mathrm{bp} 0 \mathrm{bp}$ & 1.00 (reference) & $2.19(1.44-3.33)$ & 1.00 (reference) & $2.51(1.56-4.04)$ \\
\hline $\mathrm{CC}+\mathrm{CT} / 0 \mathrm{bp} 6 \mathrm{bp}+6 \mathrm{bp} 6 \mathrm{bp}$ & 1.00 (reference) & $1.15(0.76-1.75)$ & 1.00 (reference) & $3.07(1.86-5.07)$ \\
\hline TT/0bp0bp & 1.00 (reference) & $1.18(0.39-3.50)$ & 1.00 (reference) & $2.60(0.78-8.65)$ \\
\hline TT/0bp6bp+6bp6bp & 1.00 (reference) & $5.22(1.72-15.84)$ & 1.00 (reference) & $3.81(1.19-12.22)$ \\
\hline \multicolumn{5}{|l|}{ MTHFR 677/TSER/TS 1494} \\
\hline $\mathrm{CC}+\mathrm{CT} / 3 \mathrm{R} 3 \mathrm{R} / 0 \mathrm{bp} 0 \mathrm{bp}$ & 1.00 (reference) & $2.56(1.61-4.09)$ & 1.00 (reference) & $3.03(1.74-5.25)$ \\
\hline $\mathrm{CC}+\mathrm{CT} / 3 \mathrm{R} 3 \mathrm{R} / 0 \mathrm{bp} 6 \mathrm{bp}+6 \mathrm{bp} 6 \mathrm{bp}$ & 1.00 (reference) & $1.16(0.65-2.07)$ & 1.00 (reference) & $2.61(1.30-5.26)$ \\
\hline $\mathrm{CC}+\mathrm{CT} / 2 \mathrm{R} 3 \mathrm{R}+2 \mathrm{R} 2 \mathrm{R} / 0 \mathrm{bp} 0 \mathrm{bp}$ & 1.00 (reference) & $0.92(0.32-2.61)$ & 1.00 (reference) & $1.29(0.46-3.68)$ \\
\hline $\mathrm{CC}+\mathrm{CT} / 2 \mathrm{R} 3 \mathrm{R}+2 \mathrm{R} 2 \mathrm{R} / 0 \mathrm{bp} 6 \mathrm{bp}+6 \mathrm{bp} 6 \mathrm{bp}$ & 1.00 (reference) & $1.14(0.61-2.10)$ & 1.00 (reference) & $3.55(1.71-7.37)$ \\
\hline TT/3R3R/0bp0bp & 1.00 (reference) & $0.97(0.28-3.40)$ & 1.00 (reference) & $3.97(0.95-16.58)$ \\
\hline TT/3R3R/0bp6bp+6bp6bp & 1.00 (reference) & $5.21(0.87-31.18)$ & 1.00 (reference) & $20.13(1.76-230.92)$ \\
\hline $\mathrm{TT} / 2 \mathrm{R} 3 \mathrm{R}+2 \mathrm{R} 2 \mathrm{R} / 0 \mathrm{bp} 0 \mathrm{bp}$ & 1.00 (reference) & $0.41(0.01-17.12)$ & 1.00 (reference) & $0.36(0.01-9.49)$ \\
\hline $\mathrm{TT} / 2 \mathrm{R} 3 \mathrm{R}+2 \mathrm{R} 2 \mathrm{R} / 0 \mathrm{bp} 6 \mathrm{bp}+6 \mathrm{bp} 6 \mathrm{bp}$ & 1.00 (reference) & $12.05(1.78-81.53)$ & 1.00 (reference) & $1.71(0.28-10.42)$ \\
\hline
\end{tabular}

${ }^{a}$ Models adjusted for age, gender, hypertension, diabetes mellitus and genotypes. The results of genetic factors and combinations containing lower OR values compared to total (hypertension or diabetes mellitus) were eliminated. MTHFR, methylenetetrahydrofolate reductase; TS, thymidylate synthase; TSER, TS enhancer region.

plasma folate concentrations was analyzed. Individuals with the MTHFR 677CT/1298AC genotypes and plasma folate levels $\leq 4.12 \mathrm{ng} / \mathrm{ml}$ were more likely to develop CRC. The enzyme activity of the MTHFR 677CT/AC and 677TT/AA variants is significantly lower than that of MTHFR encoded by other genotypes (16). These results suggest that MTHFR polymorphisms in the presence of low dietary folate can increase the $\mathrm{CRC}$ risk.

As the 3R allele of TSER and the 6-bp insertion allele of TS 1494 are believed to increase TS expression $(7,17,18)$, numerous studies on TS polymorphisms and cancer susceptibility have been performed. However, the results of these studies are inconclusive. A recent meta-analysis did not show any association between these TS polymorphisms (TSER and $T S$ 1494del6) and cancer risk (19). However, stratified analyses demonstrated that the TSER 2R2R genotype was associated with a significantly increased risk of cancer in the Asian population, but not in Caucasians. Similarly, the 2R2R and 2R3R genotypes were associated with reduced CRC risk in the Caucasian, but not Asian, population (19). As only two studies 
have been performed in Asian populations, these results may not be representative of all Asian populations. Several factors may contribute to the finding that $T S$ polymorphisms have differential associations with the CRC risk depending on ethnicity. First, genotype frequency may play an important role, as Caucasians have relatively high frequencies of the TSER 2R2R and TS 6bp6bp genotypes, while Asians have high frequencies of the 3R3R and 0bp0bp genotypes (11,20-23). Second, cultural factors that influence nutritional status may confound the effect of these polymorphisms on CRC susceptibility. In the present study, the $2 \mathrm{R} 2 \mathrm{R}$ variant genotype, particularly in elderly patients, non-HTN patients and non-L/N invasion patients, was associated with a significantly increased risk of CRC. Although these results indicate the TSER polymorphism in CRC development, due to the low frequency of the $2 \mathrm{R}$ allele in Korea, replicating this finding in a larger Asian sample is necessary.

$\mathrm{RFC1}$ is one of the key enzymes in folate metabolism. It is biologically plausible that $R F C l$ polymorphisms play a role in the development of cancer by altering plasma folate and homocysteine levels (8). Several studies have demonstrated an association between the $R F C 180 \mathrm{G}>\mathrm{A}$ polymorphism and cancer $(24,25)$, although no association with CRC risk has been found $(23,26,27)$. Consistent with these studies, a significant association between the RFC1 $80 \mathrm{G}>\mathrm{A}$ polymorphism and CRC risk or between the RFCl $-43 \mathrm{~T}>\mathrm{C}$ and $696 \mathrm{C}>\mathrm{T}$ polymorphisms and $\mathrm{CRC}$ were not identified. Although $\mathrm{RFCl}$ polymorphisms affect plasma folate levels, it is unlikely that they play a dominant role in folate metabolism in CRC cases. This hypothesis is supported by a previous study that RFC1 mediates the transport of naturally-occurring folate rather than folic acid, the latter representing the form of the vitamin found in supplements (28). Since folic acid is absorbed better by the body than the natural form and comprise a dominant proportion of the total folate intake, genetic variants of $R F C 1$ polymorphisms may have limited potential effect to the CRC risk. Additionally, interplay between $R F C 1$ polymorphisms and plasma folate levels did not influence CRC risk in the present study (data not shown). Thus, it is unlikely that RFC1 variants significantly affect the $\mathrm{CRC}$ risk.

Finally, a combined effect of environmental factors (HTN and DM) and folate metabolism-related polymorphisms were observed on the CRC risk. Of note, synergistic effects were identified between i) the MTHFR 677TT and MTHFR 677TT/TSER 3R3R genotypes and HTN, and ii) the MTHFR 677TT, MTHFR 677TT/TSER 3R3R and MTHFR 677TT/TSER 3R3R/TS 1494 0bp6bp+6bp6bp genotypes and DM. At present, the interactions between these genetic variants, HTN and DM are poorly understood. However, it is possible these polymorphisms could cause folate deficiency, a CRC risk factor (29), and the presence of HTN or DM, risk factors for metabolic syndrome, could accelerate CRC development (30). Thus, interactions between genetic factors causing folate deficiency and metabolic syndrome risk factors (HTN and DM) could result in increased CRC susceptibility. This hypothesis merits testing in a large, population-based study.

In conclusion, the association between seven MTHFR, TS and RFC1 polymorphisms and CRC susceptibility was investigated in a Korean population. Although these polymorphisms were not independent risk factors for CRC, a number of significant associations were identified in subsets of the population. The data suggest that the CRC risk is increased by i) $M T H F R$ polymorphisms in the presence of low plasma folate levels and ii) genetic risk factors for folate deficiency in the presence of metabolic syndrome risk factors, such as HTN and DM.

\section{Acknowledgements}

The present study was partially supported by a National Research Foundation of Korea (NRF) grant, funded by the Korean Government (NRF-2012R1A1A2007033) and partially supported by a grant of the Korea Health Technology R\&D Project through the Korea Health Industry Development Institute (KHIDI), funded by the Ministry of Health \& Welfare (grant no. HI13C1398), Republic of Korea.

\section{References}

1. Slattery ML, Potter JD, Samowitz W, Schaffer D and Leppert M: Methylenetetrahydrofolate reductase, diet and risk of colon cancer. Cancer Epidemiol Biomarkers Prev 8: 513-518, 1999.

2. Frosst P, Blom HJ, Milos R, et al: A candidate genetic risk factor for vascular disease: A common mutation in methylenetetrahydrofolate reductase. Nat Genet 10: 111-113, 1995.

3. Rozen R: Genetic predisposition to hyperhomocysteinemia: Deficiency of methylenetetrahydrofolate reductase (MTHFR). Thromb Haemost 78: 523-526, 1997.

4. Kono S and Chen K: Genetic polymorphisms of methylenetetrahydrofolate reductase and colorectal cancer and adenoma. Cancer Sci 96: 535-542, 2005.

5. Keku T, Millikan R, Worley K, Winkel S, Eaton A, Biscocho L, Martin C and Sandler R: 5,10-Methylenetetrahydrofolate reductase codon 677 and 1298 polymorphisms and colon cancer in African Americans and whites. Cancer Epidemiol Biomarkers Prev 11: 1611-1621, 2002.

6. Kawakami K, Omura K, Kanehira E and Watanabe Y: Polymorphic tandem repeats in the thymidylate synthase gene is associated with its protein expression in human gastrointestinal cancers. Anticancer Res 19: 3249-3252, 1999.

7. Mandola MV, Stoehlmacher J, Zhang W, Groshen S, Yu MC, Iqbal S, Lenz HJ and Ladner RD: A 6 bp polymorphism in the thymidylate synthase gene causes message instability and is associated with decreased intratumoral TS mRNA levels. Pharmacogenetics 14: 319-327, 2004.

8. Chango A, Emery-Fillon N, de Courcy GP, Lambert D, Pfister M, Rosenblatt DS and Nicolas JP: A polymorphism (80G- $>$ A) in the reduced folate carrier gene and its associations with folate status and homocysteinemia. Mol Genet Metab 70: 310-315, 2000.

9. Kim JW, Park HM, Choi YK, Chong SY, Oh D and Kim NK: Polymorphisms in genes involved in folate metabolism and plasma DNA methylation in colorectal cancer patients. Oncol Rep 25: 167-172, 2011.

10. Rah H, Choi YS, Jeon YJ, Choi Y, Cha SH, Choi DH, Ko JJ, Shim SH and Kim NK: Solute Carrier Family 19, member 1 (SLC19A1) polymorphisms (-43T>C, 80G $>$ A and $696 \mathrm{C}>\mathrm{T})$ and haplotypes in idiopathic recurrent spontaneous abortion in a Korean population. Reprod Sci 19: 513-519, 2012.

11. Yim DJ, Kim OJ, An HJ, Kang H, Ahn DH, Hwang SG, Oh D and Kim NK: Polymorphisms of thymidylate synthase gene 5 '- and 3'-untranslated region and risk of gastric cancer in Koreans. Anticancer Res 30: 2325-2330, 2010.

12. Chung YS, Jeon YJ, Shin DE, Min KT, Shin YS, Won KS, Koh YC, Hong SH and Kim NK: Methylenetetrahydrofolate reductase (MTHFR) and thymidylate synthase (TS) polymorphisms with osteoporotic vertebral compression fracture (OVCF) in postmenopausal Korean women. Genes Genom 34: 257-263, 2012.

13. Sheng X, Zhang Y, Zhao E, Lu S, Zheng X, Ge H and Lu W: MTHFR C677T polymorphism contributes to colorectal cancer susceptibility: Evidence from 61 case-control studies. Mol Biol Rep 39: 9669-9679, 2012. 
14. Yang Z,Zhang XF, Liu HX, Hao YS and Zhao CL: MTHFR C677T polymorphism and colorectal cancer risk in Asians, a meta-analysis of 21 studies. Asian Pac J Cancer Prev 13: 1203-1208, 2012.

15. Ma J, Stampfer MJ, Giovannucci E, Artigas C, Hunter DJ, Fuchs C, Willett WC, Selhub J, Hennekens CH and Rozen R: Methylenetetrahydrofolate reductase polymorphism, dietary interactions, and risk of colorectal cancer. Cancer Res 57: 1098-1102, 1997.

16. van der Put NM, Gabreëls F, Stevens EM, Smeitink JA Trijbels FJ, Eskes TK, van den Heuvel LP and Blom HJ: A second common mutation in the methylenetetrahydrofolate reductase gene: An additional risk factor for neural-tube defects? Am J Hum Genet 62: 1044-1051, 1998.

17. Kawakami K and Watanabe G: Identification and functional analysis of single nucleotide polymorphism in the tandem repeat sequence of thymidylate synthase gene. Cancer Res 63: 6004-6007, 2003.

18. Mandola MV, Stoehlmacher J, Muller-Weeks S, Cesarone G, Yu MC, Lenz HJ and Ladner RD: A novel single nucleotide polymorphism within the 5 'tandem repeat polymorphism of the thymidylate synthase gene abolishes USF-1 binding and alters transcriptional activity. Cancer Res 63: 2898-2904, 2003.

19. Zhou JY, Shi R, Yu HL, Zeng Y, Zheng WL and Ma WL: The association between two polymorphisms in the TS gene and risk of cancer: A systematic review and pooled analysis. Int J Cancer 131: 2103-2116, 2012.

20. Chen J, Hunter DJ, Stampfer MJ, Kyte C, Chan W, Wetmur JG, Mosig R, Selhub J and Ma J: Polymorphism in the thymidylate synthase promoter enhancer region modifies the risk and survival of colorectal cancer. Cancer Epidemiol Biomarkers Prev 12: 958-962, 2003

21. Marsh S, Collie-Duguid ES, Li T, Liu X and McLeod HL: Ethnic variation in the thymidylate synthase enhancer region polymorphism among Caucasian and Asian populations. Genomics 58: 310-312, 1999.
22. Marsh S, McKay JA, Cassidy J and McLeod HL: Polymorphism in the thymidylate synthase promoter enhancer region in colorectal cancer. Int J Oncol 19: 383-386, 2001.

23. Ulrich CM, Curtin K, Potter JD, Bigler J, Caan B and Slattery ML: Polymorphisms in the reduced folate carrier, thymidylate synthase, or methionine synthase and risk of colon cancer. Cancer Epidemiol Biomarkers Prev 14: 2509-2516, 2005.

24. de Jonge R, Tissing WJ, Hooijberg JH, Jansen G, Kaspers GJ, Lindemans J, Peters GJ and Pieters R: Polymorphisms in folate-related genes and risk of pediatric acute lymphoblastic leukemia. Blood 113: 2284-2289, 2009.

25. Wang L, Chen W, Wang J, Tan Y, Zhou Y, Ding W, Hua Z, Shen J, Xu Y and Shen H: Reduced folate carrier gene G80A polymorphism is associated with an increased risk of gastroesophageal cancers in a Chinese population. Eur J Cancer 42: 3206-3211, 2006.

26. Eklöf V, Van Guelpen B, Hultdin J, Johansson I, Hallmans G and Palmqvist R: The reduced folate carrier (RFC1) $80 \mathrm{G}>\mathrm{A}$ and folate hydrolase 1 (FOLH1) $1561 \mathrm{C}>\mathrm{T}$ polymorphisms and the risk of colorectal cancer: A nested case-referent study. Scand J Clin Lab Invest 68: 393-401, 2008.

27. Jokić M, Brčić-Kostić K, Stefulj J, Catela Ivković T, Božo L, Gamulin M and Kapitanović S: Association of MTHFR, MTR, MTRR, RFC1, and DHFR gene polymorphisms with susceptibility to sporadic colon cancer. DNA Cell Biol 30: 771-776, 2011.

28. Antony AC: The biological chemistry of folate receptors. Blood 79: 2807-2820, 1992

29. Duthie SJ: Folate and cancer: How DNA damage, repair and methylation impact on colon carcinogenesis. J Inherit Metab Dis 34: 101-109, 2011.

30. Giovannucci E: Metabolic syndrome, hyperinsulinemia, and colon cancer: A review. Am J Clin Nutr86: s836-s842, 2007. 